\title{
Stability of weak solutions to obstacle problem in Clifford analysis
}

\section{Yueming Lu* and Gejun Bao}

\section{*Correspondence:} ymlu.hit@gmail.com Department of Mathematics, Harbin Institute of Technology, Harbin, 150001, P.R. China

\begin{abstract}
This paper is concerned with the regularity properties of weak solutions to the obstacle problem for Clifford-valued functions. Our main results are a global reverse Hölder inequality and stability of the weak solutions to the obstacle problem.
\end{abstract}

Keywords: Clifford-valued function; obstacle problem; higher integrability; stability

\section{Introduction}

Let $e_{1}, e_{2}, \ldots, e_{n}$ be the standard basis of $\mathbb{R}^{n}$ with the relation $e_{i} e_{j}+e_{j} e_{i}=-2 \delta_{i j}$. For $k=$ $0,1, \ldots, n$, we denote by $C \ell_{n}^{k}=C \ell_{n}^{k}\left(\mathbb{R}^{n}\right)$ the linear space of all $k$-multivectors, spanned by the reduced products $e_{I}=e_{i_{1}} e_{i_{2}} \cdots e_{i_{k}}$, corresponding to all ordered $k$-tuples $I=$ $\left(i_{1}, i_{2}, \ldots, i_{k}\right), 1 \leq i_{1}<i_{2}<\cdots<i_{k} \leq n$. Thus, Clifford algebra $C \ell_{n}=\bigoplus_{k} C \ell_{n}^{k}$ is a graded algebra, especially $C \ell_{n}^{0}=\mathbb{R}$ and $C \ell_{n}^{1}=\mathbb{R}^{n} . \mathbb{R} \subset \mathbb{C} \subset \mathbb{H} \subset C \ell_{n}^{3} \subset \cdots$ is an increasing chain, where $\mathbb{H}$ is the Hamilton's algebra of quaternions. For $u \in C \ell_{n}, u$ can be written as

$$
u=\sum_{I} u_{I} e_{I}=\sum_{1 \leq i_{1}<\cdots<i_{k} \leq n} u_{i_{1}, \ldots, i_{k}} e_{i_{1}} \cdots e_{i_{k}}
$$

where $1 \leq k \leq n$.

The norm of $u \in C \ell_{n}$ is given by $|u|=\left(\sum_{I} u_{I}^{2}\right)^{1 / 2}$. Clifford conjugation $\overline{e_{\alpha_{1}} \cdots e_{\alpha_{k}}}=$ $(-1)^{k} e_{\alpha_{k}} \cdots e_{\alpha_{1}}$. For $u=\sum_{I} u_{I} e_{I} \in C \ell_{n}, v=\sum_{J} v_{J} e_{J} \in C \ell_{n}$,

$$
\langle u, v\rangle=\left\langle\sum_{I} u_{I} e_{I}, \sum_{J} v_{J} e_{J}\right\rangle=\sum_{I} u_{I} v_{I}
$$

defines the corresponding inner product on $C \ell_{n}$. Denote by $\operatorname{Sc}(u)$ the scalar part of $u$, the coefficient of the element $e_{0}$, and we also have $\langle u, v\rangle=\operatorname{Sc}(\bar{u} v)$.

The Dirac operator used in this paper is given by

$$
D=\sum_{j=i}^{n} e_{j} \frac{\partial}{\partial x_{j}} .
$$

Throughout this paper, we write $\mathcal{D}^{\prime}\left(\Omega, C \ell_{n}\right)$ for the space of Clifford-valued functions whose coefficients are Schwartz distributions on $\Omega$. For $p>0$, we write $L^{p}\left(\Omega, C \ell_{n}^{k}\right)$ for the space of Clifford-valued functions $u$, whose coefficients belong to the usual $L^{p}(\Omega)$ space.

\section{Springer}

(c) 2013 Lu and Bao; licensee Springer. This is an Open Access article distributed under the terms of the Creative Commons Attribution License (http://creativecommons.org/licenses/by/2.0), which permits unrestricted use, distribution, and reproduction in any medium, provided the original work is properly cited. 
It is a reflexive Banach space endowed with the norm

$$
\|u\|_{p}=\left(\int_{\Omega}|u|^{p} d x\right)^{\frac{1}{p}} .
$$

Also, $W^{1, p}\left(\Omega, C \ell_{n}^{k}\right)$ is the Sobolev space of Clifford-valued functions $u$, whose coefficients belong to $W^{1, p}(\Omega)$. It is a reflexive Banach space endowed with the norm

$$
\|u\|_{1, p}=\|u\|_{p}+\|\nabla u\|_{p}
$$

where $\nabla u=\left(\frac{\partial u}{\partial x_{1}}, \ldots, \frac{\partial u}{\partial x_{n}}\right), \frac{\partial u}{\partial x_{i}}=\sum_{I} \frac{\partial u_{I}}{\partial x_{i}} e_{I}$.

Let $\Omega$ be a bounded domain. This paper is concerned with the $A$-Dirac equation

$$
D A_{p}(x, D u)=0
$$

where $1<p<\infty, A_{p}: \Omega \times C \ell_{n} \rightarrow C \ell_{n}$ preserves the grading of the Clifford algebra and satisfies the following conditions for some constants $0<a \leq b<\infty$ :

(i) The mapping $x \rightarrow A_{p}(x, \xi)$ is measurable for all $\xi \in C \ell_{n}^{k}$.

(ii) The mapping $\xi \rightarrow A_{p}(x, \xi)$ is continuous for a.e. $x \in \Omega$.

(iii) $\left|A_{p}(x, \xi)\right| \leq b|\xi|^{p-1}$.

(iv) $\left\langle A_{p}(x, \xi)-A_{p}(x, \eta), \xi-\eta\right\rangle \geq a|\xi-\eta|^{p}$ for all $x \in \Omega$ with $\xi \neq \eta$.

Definition 1.1 [1] A Clifford-valued function $u \in W^{1, p}\left(\Omega, C \ell_{n}^{k}\right)$ for $k=0,1, \ldots, n$, is a weak solution to equation (2) if for all $\varphi \in W^{1, p}\left(\Omega, C \ell_{n}^{k}\right)$ with compact support, we have

$$
\int_{\Omega} \overline{A_{p}(x, D u)} D \varphi=0 .
$$

In $[1,2]$, Nolder explained how quasi-linear elliptic equations

$$
-\operatorname{div} A_{p}(x, \nabla u)=0
$$

arise as components of Dirac systems (2) and discussed some properties of the weak solutions to the scalar parts of equations, such as the Caccioppoli estimate and the removability theorem. In [3], Heinonen et al. studied the quasi-linear elliptic equations (4) by means of potential theory systematically. Many other mathematicians also work on properties of solutions to equations (4), such as the regularity, stability, convergence and so on, see [4-9].

This paper is organized as follows. In Section 2, some preliminary results about Cliffordvalued functions are presented. In Section 3, higher integrability of weak solutions to obstacle problem for Clifford-valued functions are obtained. Section 4 is concerned with the stability of the weak solutions to obstacle problem. For other works about Clifford analysis and Dirac equations, see [1, 2, 10-12].

\section{Preliminary results}

Lemma 2.1 $C_{0}^{\infty}\left(\Omega, C \ell_{n}^{k}\right)$ is dense in $W_{0}^{1, p}\left(\Omega, C \ell_{n}^{k}\right)$ for $1 \leq p<\infty$. 
Proof Let $u=\sum_{I} u_{I}(x) e_{I} \in W_{0}^{1, p}\left(\Omega, C \ell_{n}^{k}\right)$, then $u_{I} \in W_{0}^{1, p}(\Omega)$. Since $C_{0}^{\infty}(\Omega)$ is dense in $W_{0}^{1, p}(\Omega)$, we can find a sequence $\left\{u_{I}^{k}\right\} \subset C_{0}^{\infty}(\Omega)$ converges to $u_{I}$ in $W_{0}^{1, p}(\Omega)$. Let $u_{k}=$ $\sum_{I} u_{I}^{k} e_{I}$, then $\left\{u_{k}\right\} \subset C_{0}^{\infty}\left(\Omega, C \ell_{n}^{k}\right)$.

$$
\begin{aligned}
& \int_{\Omega}\left|u_{k}-u\right|^{p} d x+\int_{\Omega}\left|\nabla u_{k}-\nabla u\right|^{p} d x \\
& \leq \int_{\Omega}\left(\sum_{I}\left|u_{I}^{k}-u_{I}\right|^{2}\right)^{\frac{p}{2}} d x+\int_{\Omega}\left(\sum_{i=1}^{n}\left|\frac{\partial u_{k}}{\partial x_{i}}-\frac{\partial u}{\partial x_{i}}\right|^{2}\right)^{\frac{p}{2}} d x \\
& \quad \leq c(n, p) \sum_{I} \int_{\Omega}\left|u_{I}^{k}-u_{I}\right|^{p} d x+\int_{\Omega}\left(\sum_{i=1}^{n} \sum_{I}\left(\frac{\partial u_{I}^{k}}{\partial x_{i}}-\frac{\partial u_{I}}{\partial x_{i}}\right)^{2}\right)^{\frac{p}{2}} d x \\
& \quad \leq c_{1}(n, p) \sum_{I} \int_{\Omega}\left|u_{I}^{k}-u_{I}\right|^{p} d x+c_{2}(n, p) \sum_{I} \int_{\Omega}\left|\nabla u_{I}^{k}-\nabla u_{I}\right|^{p} d x .
\end{aligned}
$$

Since $\left\{u_{I}^{k}\right\}$ converges to $u_{I}$ in $W_{0}^{1, p}(\Omega)$. Then $\int_{\Omega}\left|u_{I}^{k}-u_{I}\right|^{p} d x \rightarrow 0, \int_{\Omega}\left|\nabla u_{I}^{k}-\nabla u_{I}\right| d x \rightarrow 0$. Thus,

$$
\int_{\Omega}\left|u_{k}-u\right|^{p} d x+\int_{\Omega}\left|\nabla u_{k}-\nabla u\right|^{p} d x \rightarrow 0 \quad(k \rightarrow \infty) .
$$

This means $u_{k}$ converges to $u$ in $W_{0}^{1, p}\left(\Omega, C \ell_{n}^{k}\right)$.

Here, we denote the Grassmann algebra $\bigwedge=\bigoplus_{k} \bigwedge^{k}, d$ is the exterior derivative operator, and $d^{*}$ is the formal adjoint operator. For more details about differential form, see [1]. From [13], we know that the linear mapping

$$
\lambda: e_{i_{1}} \wedge \cdots \wedge e_{i_{k}} \rightarrow e_{i_{1}} \cdots e_{i_{k}} \quad(k \geq 0)
$$

to all of $\bigwedge\left(\mathbb{R}^{n}\right)$ defines a linear isomorphism form $\bigwedge\left(\mathbb{R}^{n}\right)$ onto $C \ell_{n}\left(\mathbb{R}^{n}\right)$ independently of the choice of orthonormal basis $\left\{e_{j}\right\}$ for $\mathbb{R}^{n}$.

For a Clifford-valued function $u$, we write $u^{\sharp}$ for $\lambda^{-1} u$. Via $\lambda$, the operator $d+d^{*}$ is mapped to $D$, it means

$$
(D u)^{\sharp}=d u^{\sharp}+d^{*} u^{\sharp} \text {. }
$$

Then, if $u \in \mathcal{D}^{\prime}\left(\Omega, C \ell_{n}^{k}\right)$, there exists a constant $C$, such that

$$
\max \left\{\left|d u^{\sharp}\right|,\left|d^{*} u^{\sharp}\right|\right\} \leq C|D u| .
$$

In [4], Iwaniec gave the following Poincaré inequality for differential forms.

Theorem 2.2 For each $u^{\sharp} \in C_{0}^{\infty}\left(\Omega, \bigwedge^{k}\right)$, there exits a constant $C(n, p), 1<p<\infty$ such that

$$
\left\|\nabla u^{\sharp}\right\|_{p} \leq C(n, p)\left(\left\|d u^{\sharp}\right\|_{p}+\left\|d^{*} u^{\sharp}\right\|_{p}\right) .
$$

Combining (6), (7) and Lemma 2.1, we have the following Poincaré-Sobolev inequality. 
Proposition 2.3 For each $u \in W_{0}^{1, p}\left(\Omega, C \ell_{n}^{k}\right), 1<p<\infty$, there exists a constant $C$, such that

$$
\int_{\Omega}|\nabla u|^{p} \leq c(n, p) \int_{\Omega}|D u|^{p} d x
$$

Using the Poincaré inequality of the real function and $|\nabla| u|| \leq c(n)|\nabla u|$, we have

Corollary 2.4 For each $u \in W_{0}^{1, p}\left(\Omega, C \ell_{n}^{k}\right), 1<p<n$, there exists a constant $C$ such that

$$
\left(\int_{\Omega}|u|^{\frac{n p}{n-p}} d x\right)^{\frac{n-p}{n p}} \leq C\left(\int_{\Omega}|D u|^{p} d x\right)^{\frac{1}{p}} .
$$

Similar to the process of the Ponicaré inequality for differential forms in [14], the following inequalities for Clifford-valued function can be obtained.

Corollary 2.5 Let $u \in W_{0}^{1, s}\left(\Omega, C \ell_{n}^{k}\right), 1<s<\infty$, then, there exists a constant $C$ such that

$$
\left(f_{\Omega}|u|^{s} d x\right)^{\frac{1}{s}} \leq C|\Omega|^{\frac{1}{n}}\left(f_{\Omega}|D u|^{\frac{n s}{n+s-1}} d x\right)^{\frac{n+s-1}{n s}} .
$$

Proof If $1<s<\frac{n}{n-1}$, then $\frac{n s}{n-1} \geq\left\{s, \frac{n}{n-1}\right\}$. From the Hölder inequality and Corollary 2.4, we have

$$
\begin{aligned}
\left(f_{\Omega}|u|^{s} d x\right)^{\frac{1}{s}} & \leq\left(f_{\Omega}|u|^{\frac{n s}{n-1}} d x\right)^{\frac{n-1}{n s}} \\
& \leq C|\Omega|^{\frac{1}{n}}\left(f_{\Omega}|D u|^{\frac{n s}{n+s-1}} d x\right)^{\frac{n+s-1}{n s}} .
\end{aligned}
$$

If $s>\frac{n}{n-1}$, then

$$
\begin{aligned}
\left(\int_{\Omega}|u|^{s} d x\right)^{\frac{1}{s}} & \leq C\left(\int_{\Omega}|D u|^{\frac{n s}{n+s}} d x\right)^{\frac{n+s}{n s}} \\
& \leq C|\Omega|^{\frac{n+s}{n s}}\left(\int_{\Omega}|D u|^{\frac{n s}{n+s-1}} d x\right)^{\frac{n+s-1}{n s}} .
\end{aligned}
$$

Thus, we have

$$
\left(f_{\Omega}|u|^{s} d x\right)^{\frac{1}{s}} \leq C|\Omega|^{\frac{1}{n}}\left(f_{\Omega}|D u|^{\frac{n s}{n+s-1}} d x\right)^{\frac{n+s-1}{n s}} .
$$

\section{Higher integrability}

In this section, we will prove the higher integrability of the weak solutions to the obstacle problem $\mathcal{K}_{\psi}^{f, p}\left(\Omega, C \ell_{n}^{k}\right)$. Here, we assume that the complement of $\Omega$ satisfies the measure density condition, it means that there exists a positive constant $C>0$ such that

$$
\left|\Omega^{c} \cap B\right| \geq C|B|,
$$

where $B$ is a ball in $\mathbb{R}^{n}$. In order to prove the higher integrability, we need the following Gehring's lemma, which appeared in [5]. 
Lemma 3.1 Let $B_{0}$ be a ball in $\mathbb{R}^{n}$, and let $g, h \in L^{p}\left(B_{0}\right)$ be a nonnegative function satisfying

$$
f_{B} g^{p} d x \leq c\left[\left(f_{2 B} g d x\right)^{p}+f_{2 B} h^{q} d x\right]
$$

for all balls $B \subset 2 B \subset B_{0}, 1<p<\infty$. Then, for each $0<\sigma<1, p<s<p+\frac{p-1}{10^{n-p} 4^{n} k^{p}}$, we have

$$
\left(f_{\sigma B_{0}} g^{s} d x\right)^{\frac{1}{s}} \leq \frac{100^{n}}{\sigma^{\frac{n}{s}}(1-\sigma)^{\frac{n}{p}}}\left[\left(f_{B_{0}} g^{p} d x\right)^{\frac{1}{p}}+\left(f_{B_{0}} h^{s} d x\right)^{\frac{1}{s}}\right]
$$

Definition 3.2 For Clifford-valued functions $a=\sum_{I} a_{I}(x) e_{I}$ and $b=\sum_{I} b_{I}(x) e_{I}$, we say that $a \geq b$ in $\Omega$ if $a_{I}(x) \geq b_{I}(x)$ for a.e. $x \in \Omega$ and all ordered tuple $I$.

Let $\psi=\sum_{I} \psi_{I} e_{I}$ be a Clifford-valued function in $W^{1, p}\left(\Omega, C \ell_{n}^{k}\right)$, where $\psi_{I}: \Omega \rightarrow$ $[-\infty,+\infty) . f \in W^{1, p}\left(\Omega, C \ell_{n}^{k}\right)$ is a function, which gives the boundary. We consider the obstacle problem

$$
\mathcal{K}_{\psi}^{f, p}\left(\Omega, C \ell_{n}^{k}\right)=\left\{v \in W^{1, p}\left(\Omega, C \ell_{n}^{k}\right): v-f \in W_{0}^{1, p}\left(\Omega, C \ell_{n}^{k}\right), v \geq \psi \text { a.e. } \Omega\right\}
$$

for Clifford-valued functions. To avoid trivialities, we always assume that the set $\mathcal{K}_{\psi}^{f, p}(\Omega$, $\left.C \ell_{n}^{k}\right)$ is not empty.

Definition 3.3 We say that a function $u \in W_{\mathrm{loc}}^{1, p}\left(\Omega, C \ell_{n}^{k}\right)$ is a weak solution to the obstacle problem $\mathcal{K}_{\psi}^{f, p}\left(\Omega, C \ell_{n}^{k}\right)$, if

$$
\int_{\Omega}\left\langle A_{p}(x, D u), D v-D u\right\rangle d x \geq 0
$$

whenever $v \in \mathcal{K}_{\psi}^{f, p}\left(\Omega, C \ell_{n}^{k}\right)$.

Remark 3.4 If $u$ is a weak solution to obstacle problem (15), then $u$ holds for the scalar part of (3), i.e.,

$$
\int_{\Omega} \operatorname{Sc}(\overline{A(x, D u)} D \varphi) d x=0 .
$$

Remark 3.5 We assume $f \geq \psi$ in $\Omega$. Indeed, denote $f=\sum_{I} f_{I} e_{I}, \psi=\sum_{I} \psi_{I} e_{I}$. Then, since $u-f \in W_{0}^{1, p}\left(\Omega, C \ell_{n}^{k}\right)$,

$$
0 \leq\left(\psi_{I}-f_{I}\right)^{+} \leq\left(u-f_{I}\right)^{+}
$$

Thus, we have $\left(\psi_{I}-f_{I}\right)^{+} \in W_{0}^{1, p}(\Omega)$ and $f_{I}^{1}=\left(\psi_{I}-f_{I}\right)^{+}+f_{I} \geq \psi_{I}$ in $\Omega$. Let $f=\sum_{I} f_{I}^{1} e_{I}$, then $f \geq \psi$ in $\Omega$.

Theorem 3.6 Suppose that the complement of $\Omega$ satisfies the measure density condition (11), and let $u \in W_{\text {loc }}^{1, p}\left(\Omega, C \ell_{n}^{k}\right)$ be a weak solution to the obstacle problem (14), where $f \in$ 
Lu and Bro Advances in Difference Equations 2013, 2013:250

Page 6 of 11

http://www.advancesindifferenceequations.com/content/2013/1/250

$W^{1, p+\delta}\left(\Omega, C \ell_{n}^{k}\right)$ for some $\delta>0$. Then, there exists a positive number $\varepsilon_{0}>0$ and a constant $C$, independent of $u$, such that $|D u| \in L^{p+\varepsilon}(\Omega)$, whenever $0<\varepsilon<\varepsilon_{0}$, and

$$
\int_{\Omega}|D u|^{p+\varepsilon} d x \leq C\left[\left(\int_{\Omega}|D u|^{p} d x\right)^{1+\frac{\varepsilon}{p}}+\int_{\Omega}|D f|^{p+\varepsilon} d x\right] .
$$

Proof First, let $B_{0}$ be a ball with $\Omega \subset \frac{1}{2} B_{0}, 2 B \subset B_{0}$ be a ball such that $\Omega \cap B \neq \emptyset$ and $\eta \in C_{0}^{\infty}(2 B)$ be the cutoff function such that $\left.\eta\right|_{\bar{B}}=1,0 \leq \eta \leq 1,|\nabla \eta| \leq \frac{c(n)}{|B|^{\frac{1}{n}}}$. Denote by $D=2 B \cap \Omega$, now we test (15) with $v=u-\eta^{p}(u-f)$. Since $u-f \in W_{0}^{1, p}\left(\Omega, C \ell_{n}^{k}\right)$, then $v-f \in$ $W_{0}^{1, p}\left(\Omega, C \ell_{n}^{k}\right)$. For any $I$, we get

$$
v_{I}-\psi_{I}=\left(1-\eta^{p}\right)\left(u_{I}-\psi_{I}\right)+\eta^{p}\left(f_{I}-\psi_{I}\right) \geq 0
$$

a.e. $\Omega$. Thus, $v$ is an admissible test function, and we have

$$
\begin{aligned}
0 & \leq \int_{\Omega}\left\langle A_{p}(x, D u), D v-D u\right\rangle d x \\
& \leq \int_{D}\left\langle A_{p}(x, D u),-\eta^{p} D u+\eta^{p} D f-p \eta^{p-1} D \eta(u-f)\right\rangle d x .
\end{aligned}
$$

Since $A$ satisfies (iii), (iv), combining the Hölder inequality and the Young inequality, it follows from (18) that

$$
\begin{aligned}
a \int_{D} \eta^{p}|D u|^{p} d x \leq & b \int_{D} \eta^{p}|D u|^{p-1}|D f| d x+p \int_{D}|D u|^{p-1} \eta^{p-1}|D \eta||u-f| d x \\
\leq & b\left(\int_{D} \eta^{p}|D u|^{p} d x\right)^{\frac{p-1}{p}}\left(\int_{D} \eta^{p}|D f|^{p} d x\right)^{\frac{1}{p}} \\
& +b p\left(\int_{D} \eta^{p}|D u|^{p} d x\right)^{\frac{p-1}{p}}\left(\int_{D} \eta^{p}|u-f|^{p} d x\right)^{\frac{1}{p}} \\
\leq & \frac{b \epsilon_{1}(p-1)}{p} \int_{D} \eta^{p}|D u|^{p} d x+b c\left(p, \epsilon_{1}\right) \int_{D} \eta^{p}|D f|^{p} d x \\
& +\frac{b \epsilon_{2}(p-1)}{p} \int_{D} \eta^{p}|D u|^{p} d x+b c\left(p, \epsilon_{2}\right) \int_{D}|D \eta|^{p}|u-f|^{p} d x
\end{aligned}
$$

Choose $\epsilon_{1}, \epsilon_{2}$ such that

$$
\frac{b \epsilon_{1}(p-1)}{p}+\frac{b \epsilon_{2}(p-1)}{p} \leq \frac{a}{2} .
$$

Since $u-f \in W_{0}^{1, p}\left(\Omega, C \ell_{n}^{k}\right)$, using Lemma 2.5, we have

$$
\begin{aligned}
\int_{B \cap \Omega}|D u|^{p} d x & \leq c_{1} \int_{2 B \cap \Omega}|D f|^{p} d x+\frac{c_{2}}{|B|^{\frac{1}{n}}} \int_{2 B \cap \Omega}|u-f|^{p} d x \\
& \leq c_{1} \int_{2 B \cap \Omega}|D f|^{p} d x+\frac{c_{2}}{|B|^{\frac{1}{n}}}|2 B \cap \Omega|^{\frac{p}{n}}\left(\int_{2 B \cap \Omega}|D u-D f|^{\frac{p}{\theta}} d x\right)^{\theta},
\end{aligned}
$$


where $\theta=\frac{n+p-1}{n}>1$. By means of condition (11), we have $|2 B \cap \Omega| \leq(1-C)|2 B|$, then (19) becomes

$$
\frac{1}{|B|} \int_{B \cap \Omega}|D u|^{p} d x \leq c_{3} \frac{1}{|2 B|} \int_{2 B \cap \Omega}|D f|^{p} d x+c_{4} \frac{1}{|2 B|^{\theta}}\left(\int_{2 B \cap \Omega}|D u|^{\frac{p}{\theta}}+|D f|^{\frac{p}{\theta}} d x\right)^{\theta} .
$$

Let

$$
g(x)= \begin{cases}|D u|^{\frac{p}{\theta}}, & \text { if } x \in \Omega, \\ 0, & \text { otherwise, }\end{cases}
$$

and

$$
h(x)= \begin{cases}|D f|^{\frac{p}{\theta}}, & \text { if } x \in \Omega \\ 0, & \text { otherwise. }\end{cases}
$$

Then, the following reverse Hölder estimate

$$
\begin{aligned}
f_{B} g^{\theta} d x & \leq c_{3} f_{2 B} h^{\theta} d x+c_{4}\left(f_{2 B} g+h d x\right)^{\theta} \\
& \leq c_{5}\left(f_{2 B} g d x\right)^{\theta}+c_{6} f_{2 B} h^{\theta} d x
\end{aligned}
$$

holds when $2 B \subset B_{0}$. According to Lemma 3.1, (21) becomes

$$
\begin{aligned}
\left(f_{B}|g|^{\theta\left(1+\frac{\varepsilon}{p}\right)} d x\right)^{\frac{1}{\theta\left(1+\frac{\varepsilon}{p}\right)}} & \leq C\left[\left(f_{B_{0}}|g|^{\theta} d x\right)^{\frac{1}{\theta}}+\left(f_{B_{0}} h^{\theta\left(1+\frac{\varepsilon}{p}\right)} d x\right)^{\frac{1}{\theta\left(1+\frac{\varepsilon}{p}\right)}}\right] \\
& \leq C\left[\left(f_{\Omega}|g|^{\theta} d x\right)^{\frac{1}{\theta}}+\left(f_{\Omega} h^{\theta\left(1+\frac{\varepsilon}{p}\right)} d x\right)^{\frac{1}{\theta\left(1+\frac{\varepsilon}{p}\right)}}\right] .
\end{aligned}
$$

That is,

$$
\int_{B}|D u|^{p+\varepsilon} d x \leq C\left[\left(\int_{\Omega}|D u|^{p} d x\right)^{1+\frac{\varepsilon}{p}}+\int_{\Omega}|D f|^{p+\varepsilon} d x\right]
$$

Since $\Omega$ is a bounded domain, $\bar{\Omega}$ can be covered by a finite number of balls such that the previous inequality holds, then the estimates (17) follows immediately.

\section{Stability}

In this section, we will show that the weak solutions to obstacle problem are stable under some suitable assumptions.

Suppose that $\left\{p_{i}\right\}$ is a sequence such that $p_{i} \rightarrow p$ and for each $i=0,1, \ldots$, there is an operator $A_{p_{i}}$ satisfying (i)-(iv) for $p=p_{i}$ and for a.e. $x \in \Omega$,

$$
A_{p_{i}}(x, \xi) \rightarrow A_{p}(x, \xi)
$$


uniformly on compact subset of $\mathbb{R}^{n}$. We consider the obstacle problem

$$
\mathcal{K}_{\psi}^{f, p_{i}}\left(\Omega, C \ell_{n}^{k}\right)=\left\{v \in W^{1, p_{i}}\left(\Omega, C \ell_{n}^{k}\right): v-f \in W_{0}^{1, p_{i}}\left(\Omega, C \ell_{n}^{k}\right), v \geq \psi \text { a.e. } \Omega\right\} .
$$

Assume that $A_{p_{i}}$ preserves the grading of the Clifford algebra and converges to $A_{p}(x, \xi)$ uniformly on compact subset of $\mathbb{R}^{n}$. Suppose that $u_{i} \in W^{1, p_{i}}\left(\Omega, C \ell_{n}^{k}\right)$ is the weak solution to the following $\mathcal{K}_{\psi}^{f, p_{i}}\left(\Omega, C \ell_{n}^{k}\right)$-obstacle problem

$$
\int_{\Omega}\left\langle A_{p_{i}}\left(x, D u_{i}\right), D v-D u_{i}\right\rangle d x \geq 0
$$

for each $v \in \mathcal{K}_{\psi}^{f, p_{i}}\left(\Omega, C \ell_{n}^{k}\right)$ and $u_{0} \in W^{1, p}\left(\Omega, C \ell_{n}^{k}\right)$ is the weak solution to

$$
\int_{\Omega}\left\langle A_{p}\left(x, D u_{0}\right), D v-D u_{0}\right\rangle d x \geq 0
$$

for all $v \in \mathcal{K}_{\psi}^{f, p}\left(\Omega, C \ell_{n}^{k}\right)$.

Definition 4.1 We call a sequence $u_{j} \in L^{p}\left(\Omega, C \ell_{n}\right)$ converges weakly in $L^{p}\left(\Omega, C \ell_{n}\right)$ to $u$ if

$$
\int_{\Omega} v u_{j} d x \rightarrow \int_{\Omega} v u d x
$$

whenever $v \in L^{\frac{p}{p-1}}\left(\Omega, C \ell_{n}\right)$. Denote it by $u_{j} \rightarrow u$.

Remark 4.2 Suppose that $u_{i}$ weakly converges to $u$ in $L^{p}\left(\Omega, C \ell_{n}^{k}\right)$, then

$$
\int_{\Omega}\left\langle v, u_{i}\right\rangle d x \rightarrow \int_{\Omega}\langle v, u\rangle d x
$$

whenever $v \in W^{1, p}\left(\Omega, C \ell_{n}^{k}\right)$.

Now, we start with the main result of this section.

Theorem 4.3 Suppose that the complement of $\Omega$ satisfies the measure density condition (11). Let $p_{i}, A_{p_{i}}(x, \xi), A_{p}(x, \xi)$ be defined as described above. Let the boundary value function $f$ be in $W^{1, p+\tau}\left(\Omega, C \ell_{n}^{k}\right)$ for some $\tau>0$. Then, there is a small number $\delta_{0}$ such that the sequence $\left\{u_{i}\right\}$ of weak solutions to the obstacle problem (24) has a subsequence, which converges to the weak solution $u_{0}$ of $(25)$ in $W^{1, p+\delta}\left(\Omega, C \ell_{n}^{k}\right)$ for any $\delta<\delta_{0}$.

Before proving Theorem 4.3, we need the following lemmas.

Lemma 4.4 [6] Suppose that a bounded open set $\Omega$ satisfies (11). Let $p_{i}>1$ and $u_{i} \in$ $W^{1, p_{i}}(\Omega), i=0,1, \ldots$, and suppose that $p_{i} \rightarrow p$ and $u_{i} \rightarrow u_{0}$ a.e in $\Omega$. If $\theta \in W^{1, p+\epsilon}(\Omega)$, $\epsilon>0$ and if $u_{i}-\theta \in W_{0}^{1, p_{i}}(\Omega)$ with

$$
\int_{\Omega}\left|\nabla\left(u_{i}-\theta\right)\right|^{p_{i}} d x \leq M
$$

$i=1,2, \ldots$, where $M<\infty$ is independent of $i$, then $u_{0} \in W_{0}^{1, p}(\Omega)$. 
Lemma 4.5 Let $u_{i}$ be described in Theorem 4.3. There exists a constant $\gamma_{0}>0$ such that for each $\gamma \in\left[0, \gamma_{0}\right]$,

$$
\int_{\Omega}\left|D u_{i}\right|^{p+\frac{\gamma}{2}} d x \leq C
$$

where $i$ is sufficiently large and $C$ is independent of $i$.

Proof By virtue of Remark 3.5, we can use $f$ as a test function in (24), so that

$$
\int_{\Omega}\left\langle A_{p_{i}}\left(x, D u_{i}\right), D f-D u_{i}\right\rangle d x \geq 0 .
$$

From the structure conditions on $A_{i}$ and the Hölder inequality, it follows

$$
a \int_{\Omega}\left|D u_{i}\right|^{p_{i}} d x \leq b\left(\int_{\Omega}\left|D u_{i}\right|^{p_{i}} d x\right)^{\frac{p_{i}-1}{p_{i}}}\left(\int_{\Omega}|D f|^{p_{i}} d x\right)^{\frac{1}{p_{i}}}
$$

Then we have

$$
\int_{\Omega}\left|D u_{i}\right|^{p_{i}} d x \leq C \int_{\Omega}|D f|^{p_{i}} d x
$$

By the Hölder inequality and Young's inequality, (30) becomes

$$
\int_{\Omega}\left|D u_{i}\right|^{p_{i}} d x \leq C\left(|\Omega|+\int_{\Omega}|D f|^{p+\sigma} d x\right)
$$

when $i$ is sufficiently large and $\sigma>0$. According to Theorem 3.6, there exists a constant $\gamma_{0}>0$ such that for each $\gamma \in\left[0, \gamma_{0}\right]$,

$$
\begin{aligned}
& \left(\int_{\Omega}\left|D u_{i}\right|^{p_{i}+\gamma} d x\right)^{\frac{p_{i}}{p_{i}+\gamma}} \\
& \quad \leq C\left[\int_{\Omega}\left|D u_{i}\right|^{p_{i}} d x+\left(\int_{\Omega}|D f|^{p_{i}+\gamma} d x\right)^{\frac{p_{i}}{p_{i}+\gamma}}\right] .
\end{aligned}
$$

Since $p_{i} \rightarrow p$, choose $i$ sufficiently large such that $p-\frac{\gamma}{2} \leq p_{i} \leq p+\frac{\gamma}{2}$. Then $p+\frac{\gamma}{2} \leq p+\gamma$. So, there exists a constant $C$, such that

$$
\int_{\Omega}\left|D u_{i}\right|^{p+\frac{\gamma}{2}} d x \leq C
$$

Proof of Theorem 4.3 Let $\kappa=\frac{n}{n-1}$. Since $u_{i}-f \in W_{0}^{1, p}\left(\Omega, C \ell_{n}^{k}\right)$, we choose $i$ so large that $p+\frac{\gamma}{2} \leq \kappa p_{i}$ and $p_{i} \leq p+\frac{\gamma}{2}$, using Lemma 2.4, we get

$$
\begin{aligned}
\left\|u_{i}-f\right\|_{p+\frac{\gamma}{2}} & \leq C\left\|u_{i}-f\right\|_{\kappa p_{i}} \\
& \leq C\left\|u_{i}-f\right\|_{\frac{n p_{i}}{n-p_{i}}} \\
& \leq C\left\|D u_{i}-D f\right\|_{p_{i}} \\
& \leq C\left(\left\|D u_{i}\right\|_{p+\frac{\gamma}{2}}+\|D f\|_{p+\frac{\gamma}{2}}\right) .
\end{aligned}
$$


Implied by the Minkowski inequality that

$$
\begin{aligned}
\left\|u_{i}\right\|_{p+\frac{\gamma}{2}} & \leq\left\|u_{i}-f\right\|_{p+\frac{\gamma}{2}}+\|f\|_{p+\frac{\gamma}{2}} \\
& \leq C\left(\left\|D u_{i}\right\|_{p+\frac{\gamma}{2}}+\|D f\|_{p+\frac{\gamma}{2}}+\|f\|_{p+\frac{\gamma}{2}}\right) .
\end{aligned}
$$

Then it follows from Lemma 4.5 that

$$
\left\|u_{i}\right\|_{W^{1, p+\frac{\gamma}{2}}\left(\Omega, C \ell_{n}^{k}\right)} \leq C .
$$

Write $\delta=\frac{\gamma}{2}, u_{i}=\sum_{I} u_{I}^{i} e_{I}$, according to (33), we have $u_{i} \in W^{1, p+\delta}\left(\Omega, C \ell_{n}^{k}\right)$. So, we can extract a subsequence, still denote by $u_{I}^{i}$, such that

$$
\begin{cases}u_{I}^{i} \rightarrow u_{I}, & \text { in } W^{1, p+\delta}(\Omega), \\ u_{I}^{i} \rightarrow u_{I}, & \text { in } L^{p+\delta}(\Omega), \\ u_{I}^{i} \rightarrow u_{I}, & \text { pointwise a.e. in } \Omega .\end{cases}
$$

Then $\nabla u_{I}^{i} \rightarrow \nabla u_{I}$. Let $u=\sum_{I} u_{I} e_{I}$, then $u_{i} \rightarrow u$ in $L^{p+\delta}(\Omega) ; u_{i} \rightarrow u$ pointwise a.e. in $\Omega$. Since $\nabla u_{I}^{i} \rightarrow \nabla u_{I}$, for each $j=1,2, \ldots, n$, we have

$$
\int_{\Omega} y_{\alpha} \frac{\partial u_{I}^{i}}{\partial x_{j}} d x \rightarrow \int_{\Omega} y_{\alpha} \frac{\partial u_{I}}{\partial x_{j}} d x
$$

whenever $y_{\alpha} \in W^{1, p}(\Omega)$. Then, $\int_{\Omega} y D u_{i} d x \rightarrow \int_{\Omega} y D u d x$ for each $y \in W^{1, p}\left(\Omega, C \ell_{n}\right)$. This yields $D u_{i} \rightarrow D u$ in $L^{p}\left(\Omega, C \ell_{n}\right)$.

The next stage is to extract a further subsequence, so that $D u_{i} \rightarrow D u$ pointwise almost everywhere in $\Omega$.

Because of $u_{i}-f \in W_{0}^{1, p_{i}}\left(\Omega, C \ell_{n}^{k}\right)$ and $u_{i}-f \rightarrow u-f$, we get $u-f \in W_{0}^{1, p_{i}}\left(\Omega, C \ell_{n}^{k}\right)$ a.e. in $\Omega$, that is to say, $u \in \mathcal{K}_{\psi}^{f, p_{i}}\left(\Omega, C \ell_{n}^{k}\right)$. So,

$$
\int_{\Omega}\left\langle A_{p_{i}}\left(x, D u_{i}\right), D u-D u_{i}\right\rangle d x \geq 0 .
$$

Moreover, by the convergence assumption, we obtain

$$
A_{p_{i}}(x, D u) \rightarrow A_{p}(x, D u)
$$

almost everywhere in $\Omega$.

From this, if follows that $u_{i} \rightarrow u$ in $W^{1, p+\delta^{\prime}}\left(\Omega, C \ell_{n}^{k}\right)$ for all $\delta^{\prime}<\delta$.

At last, we will show that $u=u_{0}$. Using Lemma (4.4), we get $u-f \in W_{0}^{1, p}\left(\Omega, C \ell_{n}^{k}\right)$, then $u \in \mathcal{K}_{\psi}^{f, p}\left(\Omega, C \ell_{n}^{k}\right)$. This yields

$$
\int_{\Omega}\left\langle A_{p}\left(x, D u_{0}\right), D u-D u_{0}\right\rangle d x \geq 0
$$

On the other hand,

$$
\int_{\Omega}\left\langle A_{p_{i}}\left(x, D u_{i}\right), D u_{0}-D u_{i}\right\rangle d x \geq 0 .
$$


Let $i \rightarrow \infty$, since $u_{i} \rightarrow u$ in $W^{1, p}\left(\Omega, C \ell_{n}^{k}\right)$, we get

$$
\int_{\Omega}\left\langle A_{p}(x, D u), D u_{0}-D u\right\rangle d x \geq 0
$$

Combining (36) and (37), we have

$$
0 \leq \int_{\Omega}\left\langle A_{p}(x, D u)-A_{p}\left(x, D u_{0}\right), D u-D u_{0}\right\rangle d x \leq 0
$$

It follows that $u=u_{0}$. This proof is completed.

\section{Competing interests}

The authors declare that they have no competing interests.

\section{Authors' contributions}

All authors contributed equally in this paper. They read and approved the final manuscript.

\section{Acknowledgements}

The authors would like to thank the anonymous referees for their time and thoughtful suggestions. The research is supported by the National Science Foundation of China (\#11071048).

\section{Received: 24 May 2013 Accepted: 6 August 2013 Published: 20 August 2013}

\section{References}

1. Nolder, C: Nonlinear A-Dirac equation. Adv. Appl. Clifford Algebras 21, 429-440 (2011)

2. Nolder, C: A-harmonic equations and the Dirac operator. J. Inequal. Appl. 2010, Article ID 124018 (2010)

3. Heinonen, J, Kilpeläinen, T, Martio, O: Nonlinear Potential Theory of Degenerate Elliptic Equation. Oxford University Press, Oxford (1993)

4. Iwaniec, T, Scott, C, Stroffolini, B: Nonlinear hodge theory on manifolds with boundary. Ann. Mat. Pura Appl. CLXXVII, 37-115 (1999)

5. Iwaniec, T: The Gehring lemma. In: Quasiconformal Mappings and Analysis, pp. 181-204. Springer, Berlin (1998)

6. Li, G, Martio, O: Stability of solutions of varying degenerate elliptic equations. Indiana Univ. Math. J. 47, 873-891 (1998)

7. Ran, Q, Fang, A: Stability of solution of a class of quasilinear elliptic equations. Acta Math. Appl. Sin. 18, 461-470 (2002)

8. Li, G, Martio, O: Local and global integrability of gradients in obstacle problems. Ann. Acad. Sci. Fenn., Math. 19, 25-34 (2004)

9. Kilpeläinen, T, Malý, J: Degenerate elliptic equations with measure data and nonlinear potentials. Ann. Sc. Norm. Super. Pisa, Cl. Sci. XIX, 591-613 (1992)

10. Chen, Q, Jost, J, Li, J, Wang, G: Regularity theorems and energy identities for Dirac-harmonic maps. Math. Z. 251, 61-84 (2005)

11. Chen, Q, Jost, J, Wang, G: Nonlinear Dirac equations on Riemann surfaces. Ann. Glob. Anal. Geom. 33, 253-270 (2008)

12. Nolder, C, Ryan, J: p-Dirac equations. Adv. Appl. Clifford Algebras 19, 391-402 (2009)

13. Gilbert, J, Murray, M: Clifford Algebras and Dirac Operators in Harmonic Analysis. Cambridge University Press, Cambridge (1991)

14. Stroffolini, B: On weakly A-harmonic tensors. Stud. Math. 114, 289-301 (1995)

doi:10.1186/1687-1847-2013-250

Cite this article as: Lu and Bao: Stability of weak solutions to obstacle problem in Clifford analysis. Advances in Difference Equations 2013 2013:250. 